\title{
ANGIOSTRONGYLOSIS-RELATED RESTRICTIVE PNEUMOPATHY ASSESSED BY ARTERIAL BLOOD GAS ANALYSIS IN A DOG
}

\author{
Judit CsÖNDES ${ }^{1 *}$, Gábor MAJOROS ${ }^{2}$, Zoltán LAJOS $^{3}$, Roland PSÁDER $^{4}$, Péter VAJDOVICH ${ }^{1}$, \\ Ferenc MANCZUR ${ }^{4}$ and Ákos MÁTHÉ ${ }^{4}$ \\ ${ }^{1}$ Department of Clinical Pathology and Oncology, ${ }^{2}$ Department of Parasitology and \\ Zoology and ${ }^{4}$ Department and Clinic of Internal Medicine, Faculty of Veterinary \\ Science, Szent István University, István utca 2, H-1078 Budapest, Hungary; \\ ${ }^{3}$ DuoBakt Veterinary Laboratory, Budapest, Hungary
}

(Received 24 September 2014; accepted 29 October 2014)

Pulmonary angiostrongylosis was diagnosed by the Baermann method and larval identification from faecal and bronchoalveolar lavage samples in a fivemonth-old male mongrel dog with dyspnoea and cough. Arterial blood gas analysis indicated arterial hypoxaemia and restrictive pneumopathy. In addition to the palliative treatment, fenbendazole was administered $(50 \mathrm{mg} / \mathrm{kg} / 24 \mathrm{~h}$ per os) for 14 days. The respiratory signs subsided within a short time clinically, but serial arterial blood gas analysis demonstrated an ongoing ventilation disorder. Repeated haematology, thoracic radiography, bronchoscopy and blood gas analysis were performed to follow the course of the disease. The most severe eosinophilia was detected after the beginning of the anthelmintic therapy, and the arterial $\mathrm{pO}_{2}$ level was permanently low. Arterial blood gas analysis provided the most adequate information about the course of the pneumopathy and it greatly facilitated the patient's medical management.

Key words: Dog, bronchopneumonia, eosinophilia, pulmonary angiostrongylosis, arterial blood gas analysis, fenbendazole

Canine pulmonary angiostrongylosis (CPA) is caused by the nematode Angiostrongylus vasorum, which predominantly infects young canids (Kassai, 2003; Chapman et al., 2004; Nelson and Sellon, 2005). In Central Europe, the natural and reservoir host of $A$. vasorum is the red fox (Vulpes vulpes) (Kassai, 2003; Barutzki and Schaper, 2009). Although Hungary can be considered an endemic region, there have been few parasitological and clinical reports about CPA. Sréter et al. (2003) studied the prevalence of respiratory nematodes in cadavers of red foxes, and found A. vasorum in $5 \%$ of the cadavers examined. Majoros et al. (2010) conducted a parasitological survey of client-owned dogs in

*Corresponding author; E-mail: Csondes.Judit@aotk.szie.hu; Phone: 0036 (1) 478-4132; Fax: 0036 (1) 478-4269/8667 
Baranya County and demonstrated A. vasorum infection in the faeces of two asymptomatic dogs by the Baermann method.

The intermediate hosts of $A$. vasorum are mostly slugs and snails (Nelson and Sellon, 2005), and the final hosts become infected by ingesting the intermediate hosts containing third-stage larvae (L3). The L3 get free in the small intestines and start their migration through the intestinal wall to the mesenteric lymph nodes. Here they develop to L4 and reach the liver with the lymphatic circulation or through the vena portae. In the liver they moult again to L5 and migrate to the right side of the heart or into the pulmonary arteries via the caudal vena cava. Adult worms are located mainly in the pulmonary arteries or in the right side of the heart (Kassai, 2003; Chapman et al., 2004; Koch and Willesen, 2009). The parasites reach their final habitat on postinfection days 9 or 10 and become sexually mature. In the pulmonary capillary network, their eggs turn into first-stage larvae (L1), which enter the alveoli and migrate toward the upper airways. The L1 are expectorated or passed through the gastrointestinal tract and discharged in the faeces. The prepatent period varies between one and two months but can be longer (Patteson et al., 1993; Garosi et al., 2005; Nelson and Sellon, 2005). CPA can be subclinical, accompanied by mild, chronic symptoms or it can be a lifethreatening disease. In addition to general signs, the cardiorespiratory system, the blood coagulation and the central nervous system can all be affected (Patteson et al., 1993; Chapman et al., 2004; Garosi et al., 2005; Traversa and Guglielmini, 2008; Barutzki and Schaper, 2009). Larvae are generally identified in faecal or bronchoalveolar/transtracheal lavage samples by the Baermann method (Kassai, 2003; Chapman et al., 2004; Nelson and Sellon, 2005). Nowadays, an ELISA is also available for the detection of circulating A. vasorum antigens and/ or specific antibodies in the blood (Schnyder et al., 2011; Schucan et al., 2012).

Angiostrongylosis can be treated with benzimidazoles, levamisole or macrocyclic lactones (Patteson et al., 1993; Chapman et al., 2004; Conboy, 2004; Nelson and Sellon, 2005). Massive destruction of the worms due to anthelmintic treatment (especially when using levamisole) may elicit an anaphylactic reaction (Koch and Willesen, 2009).

Arterial blood gas analysis contributes to determining the type and severity of different respiratory disorders and helps monitor the course of pneumopathies (Haskins, 2004; Ford and Mazzaferro, 2006). Arterial hypoxaemia is diagnosed if the arterial $\mathrm{pO}_{2}$ is less than $90 \mathrm{mmHg}$ (Haskins, 2004). Diffusion impairment develops due to the thickening of the alveolar-capillary membrane in diffuse pulmonary interstitial disease or due to the loss of alveolar or capillary surface area when pulmonary interstitial oedema, fibrosis, vasculitis or emphysema develops. Diffusion impairment is usually accompanied by low arterial $\mathrm{pO}_{2}$ level and physiologic arterial $\mathrm{pCO}_{2}$ level. These laboratory alterations reveal the restrictive nature of pulmonary disease (Haskins, 2004). When Beagle dogs were infected experimentally with $A$. vasorum, moderate hypoxaemia (median $\mathrm{paO}_{2}$ 
level of $73 \mathrm{mmHg}$ and $74 \mathrm{mmHg}$, respectively) was detected 8 and 13 weeks after the inoculation (Kranjc et al., 2010).

The present study demonstrates the important role of serial arterial blood gas analysis in the clinical approach to a CPA case.

\section{Materials and methods}

An approximately 5-month-old, non-vaccinated intact male mongrel dog was admitted to the Small Animal Clinic of the Faculty of Veterinary Science, Szent István University. The dog had been found 1.5 months before its hospitalisation in a rural part of North-eastern Hungary. Dyspnoea and coughing had started 1-2 weeks before, which deteriorated despite antibiotic and glucocorticoid treatment. Besides the physical examination, additional laboratory tests and instrumental examinations indicated a respiratory disorder.

For haematology and biochemistry, blood was obtained by venipuncture from the cephalic vein. For blood gas analysis (ABL 55 Blood Gas Analyser, Denmark) the femoral artery was punctured without anaesthesia or oxygen support to obtain an arterial blood sample according to Ford and Mazzaferro (2006). Arterial samples were analysed immediately. Abdominal ultrasonography, echocardiography and plain survey thoracic radiographs were also done. Urine was collected by cystocentesis and sent for PCR examination to exclude canine distemper infection. Whole blood was taken at the evening and the modified Knott's test was performed.

Bronchoscopic examination was carried out under general anaesthesia in sternal recumbency. A flexible videobronchoscope (diameter: $5.9 \mathrm{~mm}$, length: $61 \mathrm{~cm}$, working channel diameter: $2.3 \mathrm{~mm}$; Karl Storz Germany) was used for the procedure. Bronchoalveolar lavage (BAL) was performed by wedging the bronchoscope into the smallest airways visible, followed by instillation and aspiration of $10 \mathrm{ml}$ sterile saline infusion (Salsol inf., TEVA Pharmaceutical Works Co. Ltd., Hungary) through the working channel of the endoscope with a 20-ml sterile, single-use syringe (Romed Holland, Van Oostveen Medical B.V., The Netherlands). The collected sample was divided into EDTA-K3 tubes for cytology and parasitological examination and into a transport medium with charcoal (Transport Set, Biolab Inc., Hungary) for microbiology.

For cytology testing, the cell count of the BAL sample was measured using a haematology analyser (Abacus Junior Vet 5, Diatron MI PLC, Hungary). Subsequently the sample was added to BD Falcon, 15-mL Conical Centrifuge Tubes (Thermo Fisher Scientific Inc.) and then centrifuged (Universal 32 centrifuge, Hettich Instruments, LP) with 1,600 $\mathrm{g}$ for $5 \mathrm{~min}$. Sample preparation was done as described by De Brauwer et al. (2000). The supernatant was discarded, and then the sediment was resuspended with the remaining fluid and pipetted 
onto a glass slide. Sediment smears were prepared using a technique similar to that applied for blood smears. The samples were air dried at room temperature and stained with Accustain $^{\circledR}$, Giemsa stain (Sigma-Aldrich ${ }^{\circledR}$ ). Microscopic examination was done using a binocular CX 21 Olympus microscope.

After receiving the BAL sample for microbiological evaluation, it was homogenised and spread onto Columbia agar with $5 \%$ sheep blood (V, bioMérieux, Marcy l'Etoile, France), Eosin Methylene Blue agar (EM, bioMérieux, Marcy l'Etoile, France) and Sabouraud-glucose agar with yeast extract (Sab, Bak-teszt Ltd., Hungary) according to the current guidelines (Czirók, 1999; Quinn et al., 2011). The plates were incubated at $37^{\circ} \mathrm{C}$ for $24 \mathrm{~h}$ in normal oxygen atmosphere, and then left at room temperature for another $24 \mathrm{~h}$; therefore, they were examined twice for bacterial growth. In the course of routine parasitological examination performed in the DuoBakt Laboratory, the faeces was suspended in $0.9 \% \mathrm{NaCl}$ solution and samples were prepared by flotation technique (calcium nitrate solution of $1,270 \mathrm{~g} / \mathrm{cm}^{3}$ density; Molar Ltd., Hungary). The wet mount preparations were examined under $\times 100$ and $\times 400$ magnifications, while the smears prepared with surface enrichment were examined under $\times 60$ and $\times 100$ magnifications with an optical microscope. Because of the clinical suspicion, larval isolation was also performed using the Baermann method (Foreyt and Foreyt, 2002; Kassai, 2003; Zajac and Conboy, 2007).

Larval identification from faecal and BAL samples was performed at the Department of Parasitology and Zoology, Faculty of Veterinary Science, Szent István University. The faecal samples collected from the dog were divided into small parts and baermannised in conical glasses for $24 \mathrm{~h}$. The native slimy bronchoalveolar mucous sample was spread between two glasses and visualised in a light microscope for parasitological investigation.

Hospital care included palliative support (intravenous fluid therapy, parenteral amoxicillin-clavulanic acid combination, aminophylline, bromhexine, vitamins) and anthelmintic treatment (fenbendazole in a dose of $50 \mathrm{mg} / \mathrm{kg} / 24 \mathrm{~h}$ per os) for 14 days.

\section{Results}

The poorly developed dog weighed $8.4 \mathrm{~kg}$ (body condition score: $2 / 5$ ) and was in a bad general condition. Rectal body temperature was $39.0^{\circ} \mathrm{C}$, the respiratory rate was $100 / \mathrm{min}$ and the femoral pulse frequency was $150 / \mathrm{min}$. A moderate amount of serous nasal discharge was continuously dripping from both nostrils. The buccal mucosa was slightly pale and the capillary refill time was $1.5 \mathrm{sec}$. The pharyngeal mucosa was reddened and covered with white foamy discharge. Petechiae or ecchymosis were not detected. The mandibular lymph nodes were slightly enlarged but the other regional lymph nodes had normal size. At rest the dog suffered from moderately severe mixed-type tachydyspnoea 
which deteriorated during motion and excitement but cyanosis was absent. The recurrent productive cough was easy to provoke and it ended in retching. Above the trachea mild narrowing sounds could be heard during auscultation. Above the middle and the dorsal parts of the thoracic cavity intensified bronchial respiratory sounds and mild crackling sounds were auscultated symmetrically. Over the ventral third of the thorax weak alveolar respiratory sounds were detected. The heart rate was $150 /$ min without arrhythmia or cardiac murmur. The abdominal cavity was slightly distended, but the undulation test was negative. During deep palpation of the abdomen sensitive intestinal loops were detected. The dog was weak but conscious. The locomotor and neurological systems were found intact by physical examination.

Haematological examination revealed normocytic normochromic anaemia and leukocytosis with marked eosinophilia. The platelet count was physiological (Table 1). Alkaline phosphatase activity was moderately elevated, while other biochemical parameters were within the reference interval. The clotting times (APTT, PTT) were physiological.

The orthogonal thoracic radiographs revealed a diffuse non-structured interstitial pulmonary infiltrate showing a patchy distribution over the whole lung area. A marked peribronchial infiltrate was also apparent around the large bronchi. The entire trachea had decreased diameter (the tracheal diameter/thoracic inlet ratio was 0.13 ). The cardiac silhouette was unremarkable (Fig. 1).

During abdominal ultrasonography enlarged mesenteric lymph nodes and thickened intestinal walls were detected. PCR examination of the urine for canine distemper virus infection was negative. On echocardiographic examination, the heart had a physiological shape and the cardiac chambers had normal diameters and wall thicknesses. The shape and movement of the interventricular septum, just like the diameter of the main pulmonary artery and the shape and movement of the pulmonary valves, were also normal. There was no detectable tricuspid or pulmonary regurgitation during the Doppler interrogation of these structures. Based on these findings, pulmonary hypertension was excluded. The modified Knott's test was negative.

By bronchoscopy, a small amount of yellowish, sticky mucopurulent discharge was detected in the trachea and in the cavities of the major lobular bronchi (Fig. 2). Cytological evaluation of the bronchoalveolar mucus revealed that the specimen was rich in cells (Fig. 3). The total nucleated cell count was $524 \times$ $10^{9} / 1$. Ciliated (25\%) and non-ciliated (15\%) columnar epithelial cells were found in the sample. Some ciliated (10\%) and non-ciliated (7\%) cuboidal epithelial cells and goblet cells $(2-5 \%)$ were also visible. Alveolar macrophages were present in a ratio of $26-30 \%$. Neutrophil granulocytes, generally segmented forms, appeared in 6-8\%. Eosinophil granulocytes were also visible in moderate numbers 
Table 1

Haematology results

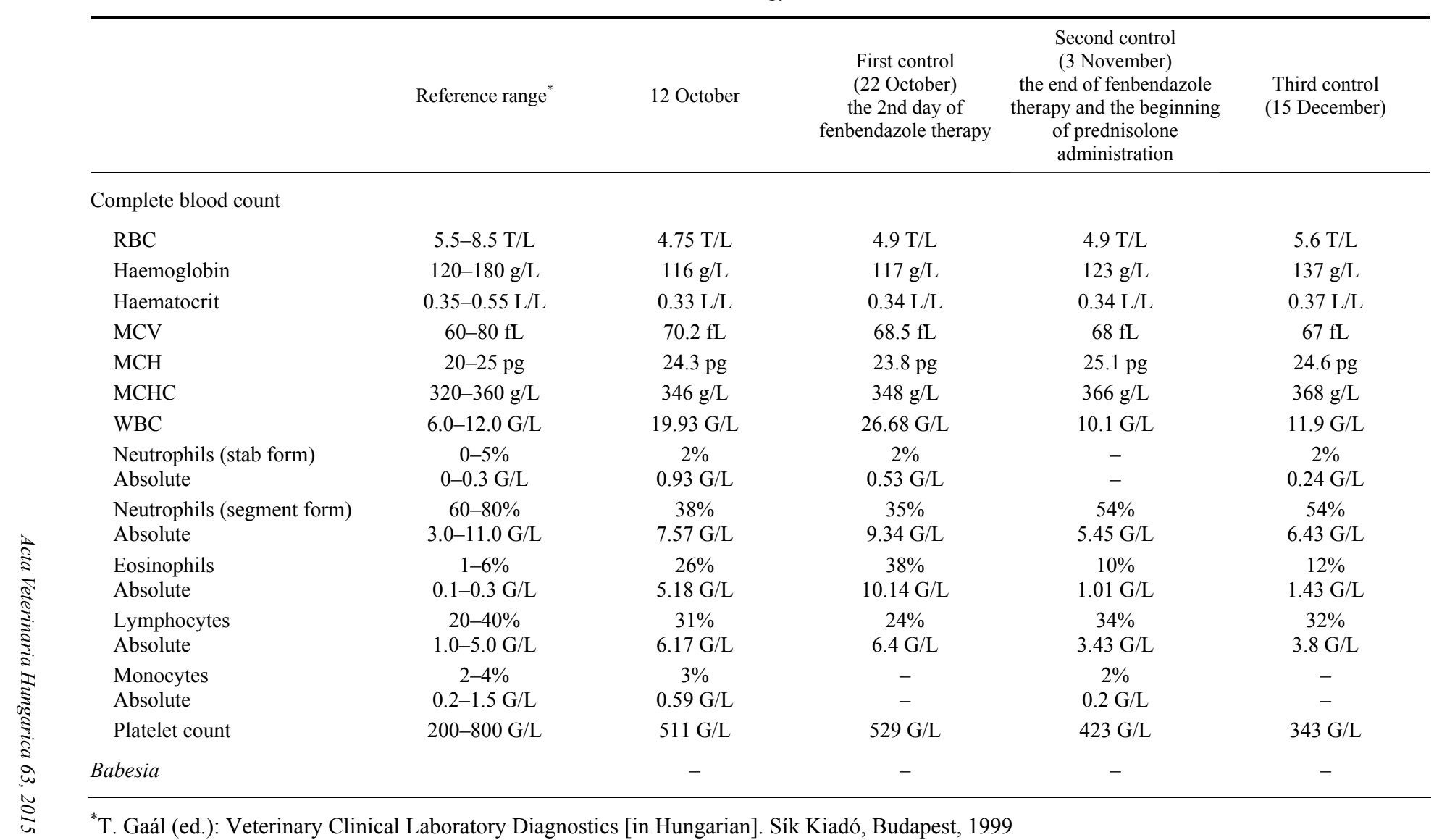


$(10-12 \%)$. In one of the smears a lungworm larva (length $360.4 \mu \mathrm{m}$, width varying between 12.9-17.9 $\mu \mathrm{m}$ with a cephalic button at the apical end) was detected (Fig. 4). This was coiled from one end. It had a cephalic button at the apical end and the tail terminated in a sinus wave curve ('severe kink') with a wanly visible dorsal spine. Based on the cytological examination a subacute proliferative inflammation and lungworm infection were demonstrated. The microbiological examination of the BAL sample was negative.

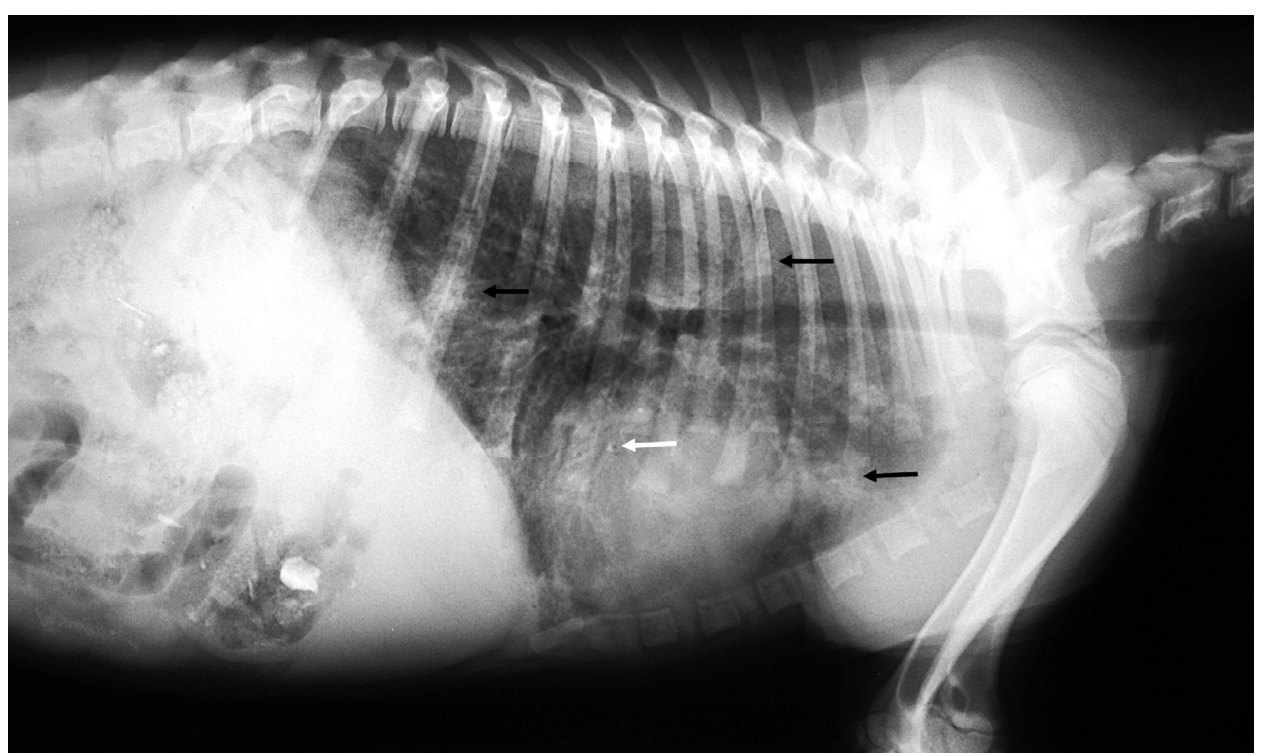

Fig. 1. The thoracic X-ray revealed patchy distribution of areas with diffuse interstitial pulmonary pattern (black arrows) and pronounced peribronchial infiltration around the large bronchi (white arrow)

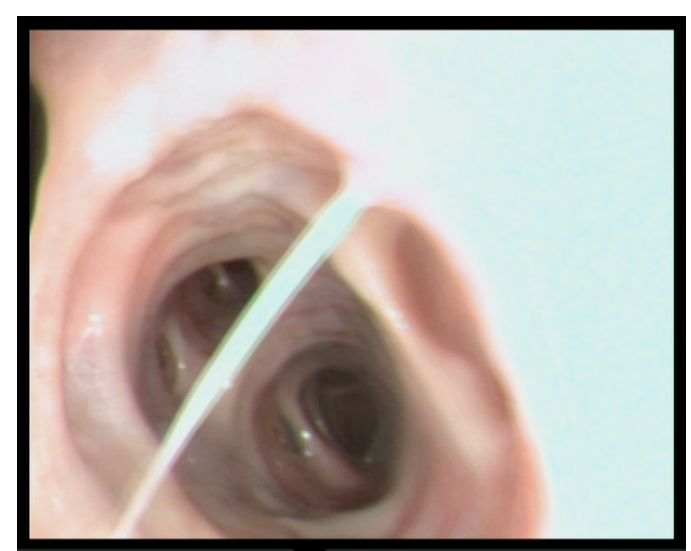

Fig. 2. The appearance of bronchi during endoscopy. Note the mucosal irregularity and the slightly yellowish colour of the secretion 


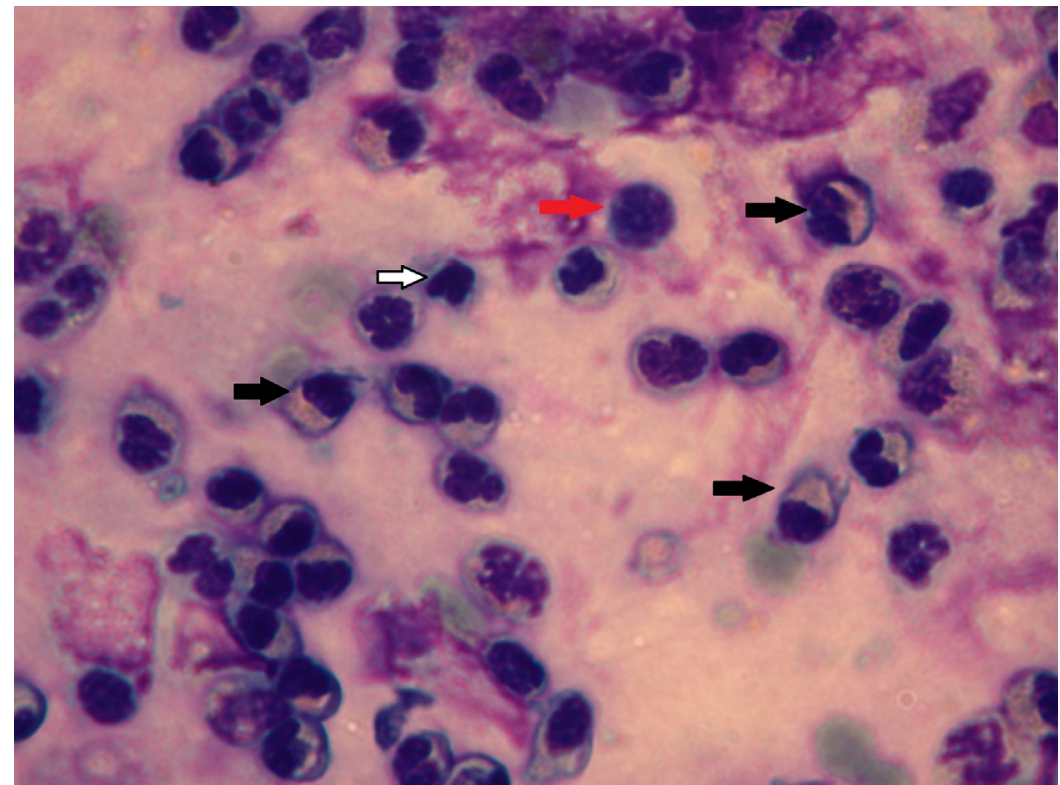

Fig. 3. Principally eosinophil (full black arrow) and neutrophil (black frame arrow) granulocytes are seen, with the occasional presence of small lymphocytes (red arrow), in the bronchoalveolar lavage sample by cytological evaluation

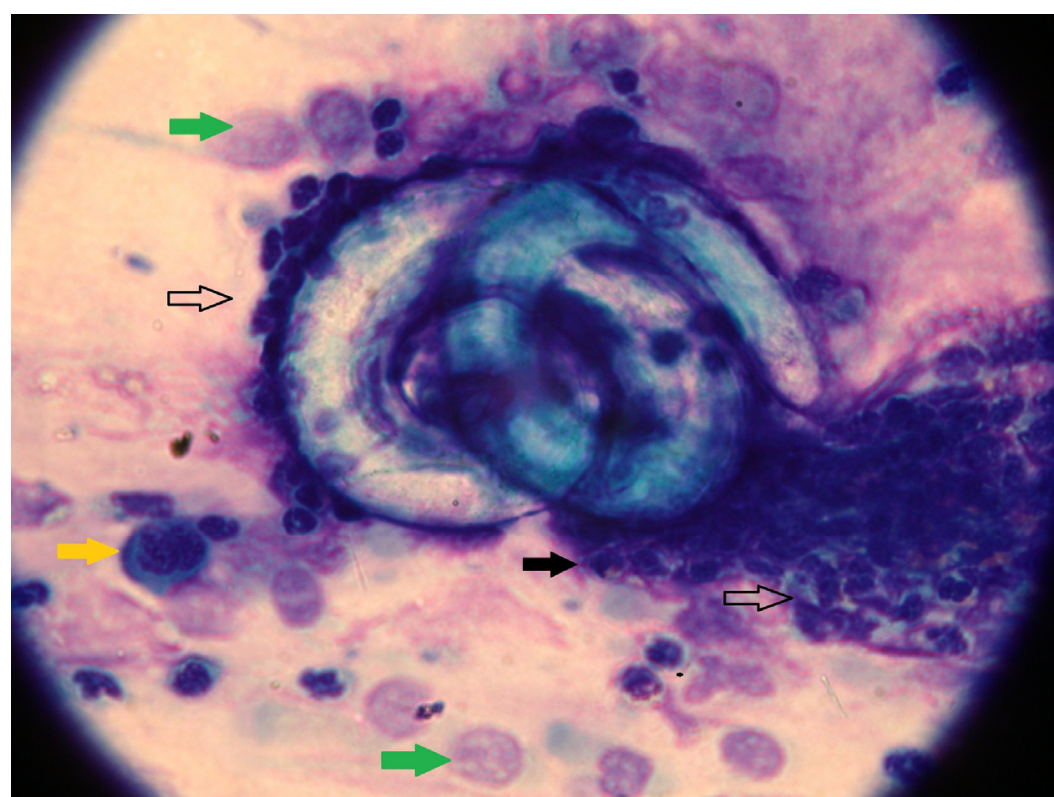

Fig. 4. Principally cuboidal epithelial cells (green arrow), alveolar macrophages (orange arrow), neutrophil (black frame arrow) and eosinophil granulocytes (full black arrow) and the lungworm larva are seen in the bronchoalveolar lavage sample 
The parasitological investigation of BAL samples revealed some living and dead forms of larval nematodes; however, these free larvae and eggshells with contorted larvae within them were not suitable for larval identification. In the faecal sample only free and living larvae could be detected. The free larvae were slightly bigger than the unhatched ones, and they contained much more granules. Their length reached $260-360 \mu \mathrm{m}$ and the refractive granules accumulated mainly in the rear half of the body. The more or less clear oesophagus and the slightly darker intestine were of equal size, as their junction could be detected at the middle of the total length. The caudal third of the oesophagus was slightly swollen in front of the junction with the intestine. The head of larvae had a button-like protuberance on it. The larvae had a tapered end of tail which narrowed unequally, moving the tip of the tail slightly towards the dorsal side (Fig. 5). Only 12 specimens of parasitic nematode larvae were found on the bottom of the glasses at the end of the isolation period.

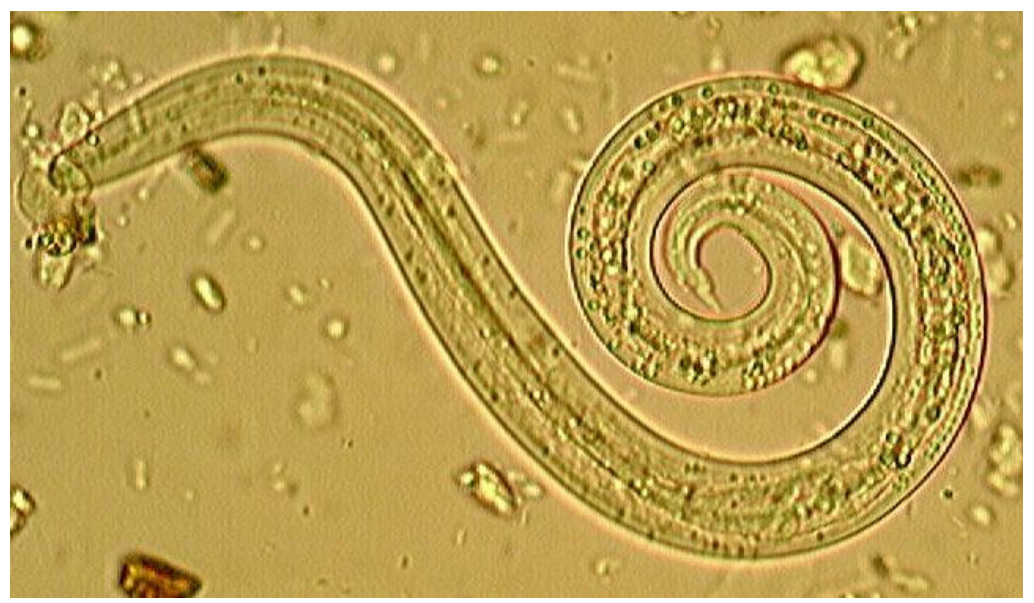

Fig. 5. Angiostrongylus vasorum larva detected by the Baermann technique

Arterial blood gas analysis revealed a mild ventilation disorder at the time of admission to the hospital. The arterial $\mathrm{pCO}_{2}\left(\mathrm{paCO}_{2}\right)$ value was within the reference range $(39.9 \mathrm{mmHg})$, while the arterial $\mathrm{pO}_{2}\left(\mathrm{paO}_{2}\right)$ level was moderately low $\left(75.6 \mathrm{mmHg}\right.$ ). The $\mathrm{PaO}_{2} / \mathrm{FiO}_{2}$ ratio was 360 (Table 2). Based on the results of analysis, restrictive pneumopathy with arterial hypoxaemia was diagnosed. On the second day of fenbendazole treatment, haematology and blood gas analysis were repeated. Eosinophilia was getting more severe and the arterial hypoxaemia was still present: the $\mathrm{paO}_{2}$ level was low $(84.4 \mathrm{mmHg})$ and the $\mathrm{paCO}_{2}$ value remained within the physiologic range $(38.6 \mathrm{mmHg})$. The $\mathrm{PaO}_{2} / \mathrm{FiO}_{2}$ ratio was 401.9 (Table 2). 
Table 2

Results of the arterial blood gas analyses

\begin{tabular}{lccccc}
\hline & $\begin{array}{c}\text { Reference } \\
\text { range }\end{array}$ & 12 October & $\begin{array}{c}\text { First } \\
\text { control } \\
\text { (22 October) }\end{array}$ & $\begin{array}{c}\text { Second } \\
\text { control } \\
\text { (3 November) }\end{array}$ & $\begin{array}{c}\text { Third } \\
\text { control } \\
\text { (15 December) }\end{array}$ \\
\hline $\mathrm{pH}$ & $7.35-7.45$ & 7.39 & 7.41 & 7.39 & 7.39 \\
$\mathrm{pCO}_{2}(\mathrm{mmHg})$ & $36-44$ & 39.9 & 38.6 & 38.5 & 36.6 \\
$\mathrm{pO}_{2}(\mathrm{mmHg})$ & $90-100$ & 75.6 & 84.4 & 77.6 & 92.3 \\
$\mathrm{PaO}_{2} / \mathrm{FiO}_{2}$ & 476 & 360 & 401.9 & 369.5 & 439.5 \\
$\mathrm{HCO}_{3}(\mathrm{mmol} / \mathrm{l})$ & $20-25$ & 22.8 & 23.7 & 22.8 & 21.5 \\
$\mathrm{ABE}^{+}(\mathrm{mmol} / \mathrm{l})$ & \pm 3.5 & -0.7 & 0.3 & -0.7 & -1.6 \\
$\mathrm{~K}^{+}(\mathrm{mmol} / \mathrm{l})$ & $3.5-5.5$ & 4.2 & 4.2 & 4.2 & 4.1 \\
$\mathrm{Na}^{+}(\mathrm{mmol} / \mathrm{l})$ & $135-155$ & 144 & 145 & 146 & 145 \\
$\mathrm{Ca}^{2+}(\mathrm{mmol} / \mathrm{l})$ & $0.8-1.5$ & 1.02 & 1.12 & 1.17 & 1.25 \\
\hline
\end{tabular}

${ }^{*}$ Ford, R. B. and Mazzaferro, E. M. (2006): Kirk and Bistner's Handbook of Veterinary Procedures and Emergency Treatment. Elsevier, St. Louis, pp. 508-509

As a result of intensive care, the patient's condition markedly ameliorated. Tachydyspnoea and coughing improved, the respiratory sounds above the chest were still harsh but less than before, and the crackling noises ceased. Fenbendazole therapy $(50 \mathrm{mg} / \mathrm{kg} / 24 \mathrm{~h}$ per os altogether for 14 days) combined with palliative support (oral administration of aminophylline, multivitamin and probiotics) was continued at home.

On the 14th day of fenbendazole therapy the dog's general condition improved and the respiratory signs ameliorated. The faecal analysis (samples were collected on three consecutive days) was negative by the Baermann technique. On the orthogonal thoracic radiographs the lungs had the same pulmonary pattern but the general radiopacity of the thorax decreased (Fig. 6). Repeated echocardiography did not detect pulmonary hypertension. During repeated bronchoscopy and BAL sampling the mucosal surface of the lower respiratory tract was covered with a small amount of sticky discharge and the mucosa was slightly irregular, but amelioration was demonstrated as compared to the first endoscopic findings. Baermann isolation from the BAL sample was negative. By cytological evaluation the BAL sample was slightly cellular, with a slightly increased mucous production and normal epithelial production as signs of a chronic inflammatory process.

Repeated complete blood count revealed that the eosinophilia moderated (Table 1) and in the actual arterial blood gas sample arterial hypoxaemia still persisted. The $\mathrm{paO}_{2}$ level was low $(77.6 \mathrm{mmHg})$ and the $\mathrm{paCO}_{2}$ value remained in the normal range $\left(38.5 \mathrm{mmHg}\right.$ ). The $\mathrm{PaO}_{2} / \mathrm{FiO}_{2}$ ratio was 369.5 (Table 2). 


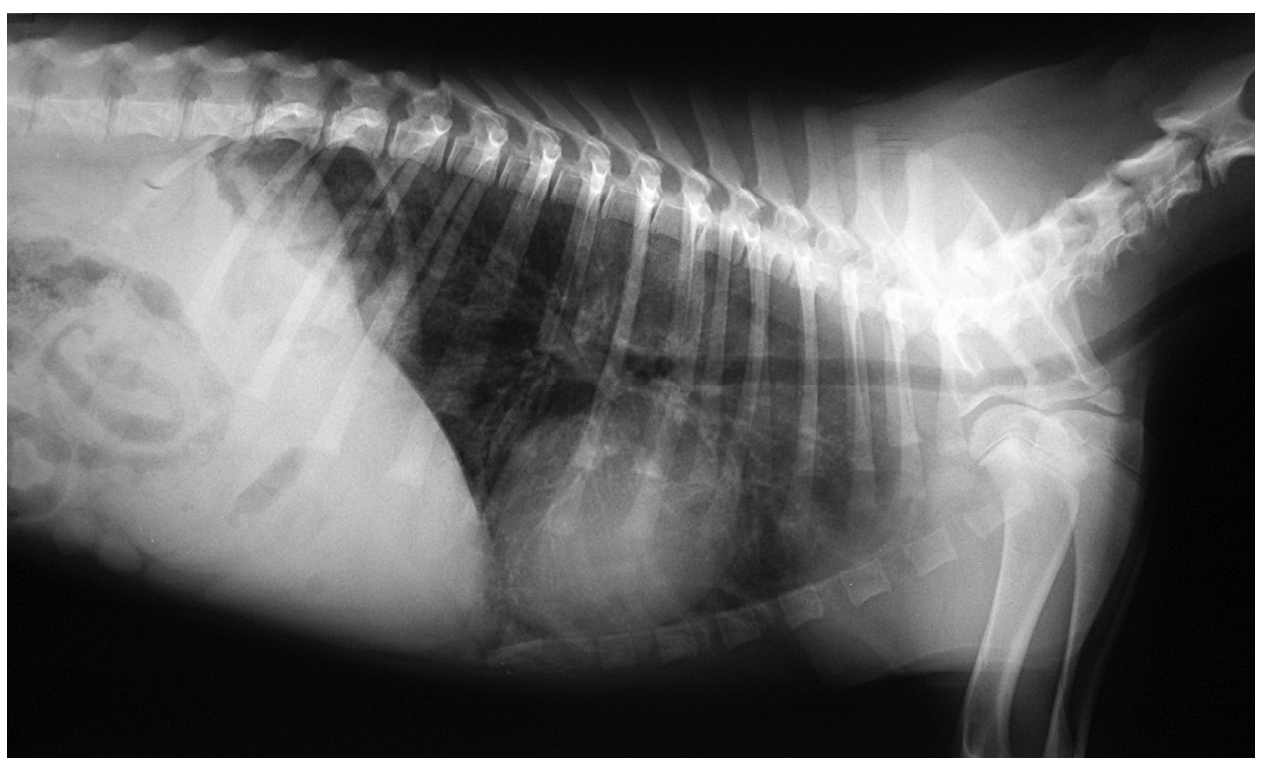

Fig. 6. The control thoracic radiograph. Note amelioration of the radiopacity as compared to the first X-ray picture

Fenbendazole administration was terminated. Prednisolone $(0.5 \mathrm{mg} / \mathrm{kg}$ per os in the mornings) was started with $\mathrm{H}_{2}$-receptor blocker (famotidine, $0.5 \mathrm{mg} / \mathrm{kg}$ / $12 \mathrm{~h}$ per os) to prevent the supposed ongoing inflammation-related fibrosis in the lungs. A local inflammatory process triggered by the dying/dead lungworms in the pulmonary tissue was suspected by this time, because the $\mathrm{paO}_{2}$ level and $\mathrm{PaO}_{2} / \mathrm{FiO}_{2}$ ratio were still decreased with elevated eosinophil numbers in the blood.

The final control examination was two months after the dog was released from the Intensive Care Unit. By that time the dog was asymptomatic, with no pathological respiratory sounds being detected over the thoracic cavity. Elevated numbers of eosinophil granulocytes were present in the blood (Table 1) but the level of $\mathrm{paO}_{2}$ was physiological $\left(92.3 \mathrm{mmHg}\right.$ ) with an almost normal $\mathrm{PaO}_{2} / \mathrm{FiO}_{2}$ ratio of 439.5 (Table 2). Bronchodilator therapy was discontinued and administration of prednisolone was gradually tapered off.

\section{Discussion}

Bronchopneumonia and eosinophilia related to A. vasorum infection were diagnosed in our patient. Diseases which can potentially cause respiratory disorders and/or eosinophilia in a young, non-vaccinated dog were considered. There are many viral respiratory infections which can cause pneumonia (canine distemper virus; canine adenovirus type 2 , canine respiratory coronavirus, canine 
influenza virus, canine parainfluenza virus). Canine distemper was excluded by PCR from a urine sample because of its clinical importance. Bacterial infections (e.g. Bordetella bronchiseptica, Klebsiella spp., Pasteurella multocida, Pseudomonas spp., Streptococcus spp., Staphylococcus spp., Mycoplasma spp., Chlamydia spp.) of the respiratory tract or pulmonary aspergillosis were not confirmed by microbiological culture from the BAL sample. Heartworm disease was excluded by echocardiography and modified Knott's test. Faecal analysis did not reveal Toxocara or Ancylostoma infestation which can be accompanied by respiratory signs. An A. vasorum larva was revealed by larval isolation and distinguished from other respiratory parasites (e.g. above all Crenosoma vulpis, but also Oslerus osleri, Filaroides spp. and Capillaria aerophila). There are several non-infectious diseases which can cause respiratory disorders and/or eosinophilia (e.g. eosinophilic bronchopneumopathy, allergy, paraneoplastic disorder, primary ciliary dyskinesia, eosinophilic leukaemia, mastocytoma, panostitis, eosinophilic myositis, hypoadrenocorticism, lead toxicosis).

The effect of anthelmintic therapy was followed up using several additional laboratory methods and diagnostic imaging techniques: Baermann test, haematology, arterial blood gas analysis, echocardiography, bronchoscopy and thoracic radiographs. Repeated Baermann examination recommended by Koch and Willesen (2009) and Paradies et al. (2013) was carried out from a faecal sample on the 14th day of fenbendazole therapy and about 6 weeks after the end of fenbendazole treatment, and they did not reveal persistent $A$. vasorum infection. We found that fenbendazole was effective and safe for treating pulmonary angiostrongylosis.

In accordance with the echocardiographic results found in the experimental work of Kranjc et al. (2010), neither abnormalities in cardiac morphology and function nor pulmonary hypertension were detected in our patient.

Determination of the level of eosinophil granulocytes in the blood, thoracic radiographs and physical examination provided useful information about the course of the disease, but with serial blood gas analysis the inflammatory process and its effect on ventilation could be followed more precisely (Fig. 7). From the arterial blood gas levels the restrictive character of the pathological process was concluded, which is presumably caused by the affected pulmonary parenchyma (Haskins, 2004). The lowest $\mathrm{paO}_{2}$ level could be explained by the local inflammatory process triggered by the dying/dead lungworms in the pulmonary tissue. The ongoing pulmonary inflammation might have caused pulmonary fibrosis. To prevent this pathological process, glucocorticoid was administered in an anti-inflammatory dose. After 6 weeks of prednisolone administration the repeated arterial blood gas analysis showed a pronounced improvement (Table 2).

Serial arterial blood gas analysis was an adequate and sensitive method for following the course of restrictive pneumopathy associated with angiostrongylosis, and it substantially contributed to the patient's successful medical management. 


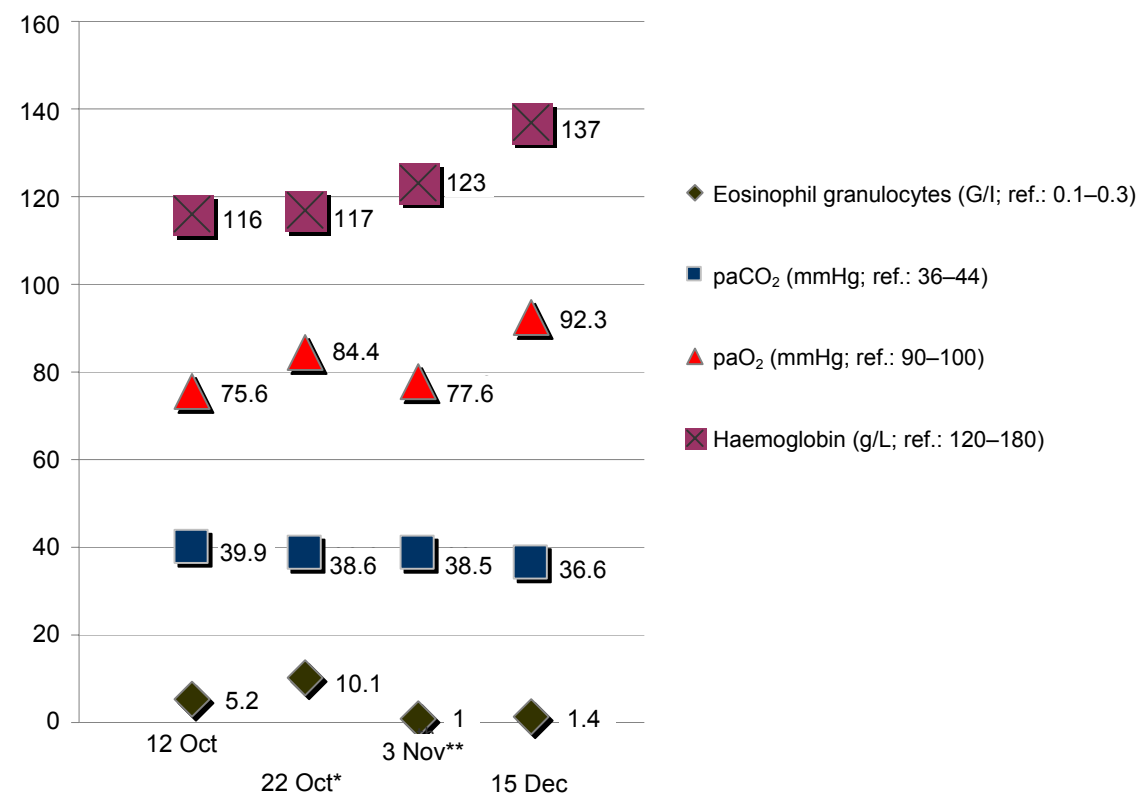

Fig. 7. Changes of several laboratory parameters during the course of pulmonary angiostrongylosis. Explanations: ${ }^{*}$ day 2 of fenbendazole therapy; ${ }^{* *}$ the end of fenbendazole treatment and the beginning of prednisolone administration

\section{Acknowledgements}

The authors thank Zoltán Dudás-Györki and Csaba Hetyey for their help with abdominal ultrasonography, Attila Arany-Tóth and Péter Csébi for evaluation of the thoracic radiographs, Olga Jacsó for parasitological evaluation of the blood sample, and Zoltán Demeter for the PCR examination. The authors wish to acknowledge the contribution of the nursing staff and the veterinarian laboratory technicians. Thanks are also due to the local veterinarian, Lóránd Bartos and the owners of the dog for their helpful contribution.

\section{References}

Barutzki, D. and Schaper, R. (2009): Natural infections of Angiostrongylus vasorum and Crenosoma vulpis in dogs in Germany (2007-2009). Parasitol. Res. 105, 39-48.

Chapman, P. S., Boag, A. K., Guitian, J. and Boswood, A. (2004): Angiostrongylus vasorum infection in 23 dogs (1999-2002). J. Small Anim. Pract. 45, 435-440.

Czirók, É. (ed.) (1999): Clinical and Epidemiological Bacteriology [in Hungarian]. Melania, Budapest, Hungary. $820 \mathrm{pp}$.

Conboy, G. (2004): Natural infections of Crenosoma vulpis and Angiostrongylus vasorum in dogs in Atlantic Canada and their treatment with milbemycin oxime. Vet. Rec. 155, 16-18. 
De Brauwer, E. I., Jacobs, J. A., Nieman, F., Bruggeman, C. A., Wagenaar, S. S. and Drent, M. (2000): Cytocentrifugation conditions affecting the differential cell count in bronchoalveolar lavage fluid. Anal. Quant. Cytol. Histol. 22, 416-422.

Ford, R. B. and Mazzaferro, E. M. (2006): Diagnostic and Therapeutic Procedures. In: Kirk and Bistner's Handbook of Veterinary Procedures and Emergency Treatment. Elsevier, St. Louis. pp. 508-509.

Foreyt, W. J. and Foreyt, B. (2002): Veterinary Parasitology Reference Manual. Fifth edition. Blackwell Publishing, Oxford. 235 pp.

Gaál, T. (ed.) (1999): Veterinary Clinical Laboratory Diagnostics [in Hungarian]. Sík Kiadó, Budapest.

Garosi, L. S., Platt, S. R., McConnell, J. F., Wray, J. D. and Smith, K. C. (2005): Intracranial haemorrhage associated with Angiostrongylus vasorum infection in three dogs. J. Small Anim. Pract. 46, 93-99.

Haskins, S. C. (2004): Interpretation of blood gas measurements, In: King, L. G. (ed.) Textbook of Respiratory Disease in Dogs and Cats. Elsevier, Oxford. pp. 181-193.

Kassai, T. (2003): Lungworm infections of predators. In: Kassai, T. (ed.) Helminthology [in Hungarian]. Medicina Könyvkiadó, Budapest, Hungary. pp. 154-156.

Koch, J. and Willesen, J. L. (2009): Canine pulmonary angiostrongylosis: An update. Vet. J. 179, 348-359.

Kranjc, A., Schnyder, M., Dennler, M., Fahrion, A., Makara, M., Ossent, P., Morgan, J., Deplazes, P. and Glaus, T. M. (2010): Pulmonary artery thrombosis in experimental Angiostrongylus vasorum infection does not result in pulmonary hypertension and echocardiographic right ventricular changes. J. Vet. Intern. Med. 24, 855-862.

Majoros, G., Fukár, O. and Farkas, R. (2010): Autochthonous infection of dogs and slugs with Angiostrongylus vasorum in Hungary. Vet. Parasitol. 174, 351-354.

Nelson, O. L. and Sellon, R. K. (2005): Pulmonary parenchymal disease. In: Ettinger, S. J. and Feldman, E. C. (eds) Textbook of Veterinary Internal Medicine. Elsevier, Philadelphia. pp. $1254-1258$.

Paradies, P., Schnyder, M., Capogna, A., Paolo Lia, R. and Sasanelli, M. (2013): Canine angiostrongylosis in naturally infected dogs: clinical approach and monitoring of infection after treatment. Sci. World J. 29, 1-8.

Patteson, M. W., Gibbs, C., Wotton, P. R. and Day, M. J. (1993): Angiostrongylus vasorum infection in seven dogs. Vet. Rec. 133, 565-570.

Quinn, P. J., Markey, B. K., Leonard, F. C., FitzPatrick, S. E., Fanning, S. and Hartigan, P. J. (2011): Veterinary Microbiology and Microbial Disease. Second edition. Blackwell Publishing, Oxford. $928 \mathrm{pp}$.

Schnyder, M., Tanner, I., Webster, P., Barutzki, D. and Deplazes, P. (2011): An ELISA for sensitive and specific detection of circulating antigen of Angiostrongylus vasorum in serum samples of naturally and experimentally infected dogs. Vet. Parasitol. 179, 152-158.

Schucan, A., Schnyder, M., Tanner, I., Barutzki, D., Traversa, D. and Deplazes, P. (2012): Detection of specific antibodies in dogs infected with Angiostrongylus vasorum. Vet. Parasitol. 185, 216-224.

Sréter, T., Széll, Z., Marucci, G., Pozio, E. and Varga, I. (2003): Extraintestinal nematode infections of red foxes (Vulpes vulpes) in Hungary. Vet. Parasitol. 4, 329-334.

Traversa, D. and Guglielmini, C. (2008): Feline aelurostrongylosis and canine angylostrongylosis: A challenging diagnosis for two emerging verminous pneumonia infections. Vet. Parasitol. 157, 163-174.

Zajac, A. M. and Conboy, G. A. (2007): Veterinary Clinical Parasitology. Blackwell Publishing, Oxford, $320 \mathrm{pp}$. 